\title{
Predictors of inducible ischemia with radionuclide stress testing: Choosing the right patients when the patients are changing
}

\author{
Hayan Jouni, $M D,{ }^{a}$ and Raymond J. Gibbons, $M D^{a}$ \\ a Department of Cardiovascular Medicine, Mayo Clinic, Rochester, MN
}

Received Sep 27, 2021; accepted Sep 28, 2021

doi: $10.1007 / \mathrm{s} 12350-021-02853-\mathrm{x}$

\section{See related article, pp. 2839-2849}

Over the past few years, there has been increasing evidence indicating a decreased frequency of abnormal single photon emission computed tomography myocardial perfusion imaging tests (SPECT). The presence, extent, and severity of inducible myocardial ischemia have all decreased in patients with a history of coronary artery disease (CAD) as well as in patients without a known history of CAD. ${ }^{1-3}$ As the yield of SPECT has declined, there is an increasing need to identify patient cohorts where SPECT will have the greatest impact on clinical management.

In this issue of the Journal of Nuclear Cardiology, Rozanski and colleagues are to be commended for performing a detailed evaluation of factors that could predict abnormal ischemic SPECT studies. ${ }^{4}$ They included SPECT studies performed at Cedars-Sinai Hospital between 2004 and 2017. In a multivariable analysis, they demonstrate that history of CAD, presence of typical angina, and reduced resting left ventricular ejection fraction $(\mathrm{LVEF}<55 \%)$ were the strongest predictors of inducible myocardial ischemia. Other predictors included male sex, age, pharmacologic stress/ low exercise capacity, diabetes, abnormal electrocardiogram (ECG), and history of hyperlipidemia. The authors conclude that these predictors can help identify patients with a higher likelihood of inducible ischemia

Reprint requests: Hayan Jouni, MD, Department of Cardiovascular Medicine, Mayo Clinic, 200 First Street SW, Rochester, MN 55905; Jouni.Hayan@mayo.edu

J Nucl Cardiol 2022;29:2850-2.

$1071-3581 / \$ 34.00$

Copyright (C) 2021 American Society of Nuclear Cardiology. and thereby make SPECT more cost-effective. They also propose that future iterations of appropriate use criteria (AUC) employ revised algorithms for estimating CAD likelihood.

However, we would urge caution in the application of these data to contemporary practice. First, as noted above, the decline of abnormal SPECT studies over the past 30 years has been reported from multiple large medical centers. ${ }^{2,3,5}$ Although Rozanski and colleagues rigorously evaluated the predictors of ischemia between 2004 and 2017, they did not address the potential impact of the timing of testing on their results. To what degree do these predictors (typical angina, reduced LVEF, etc.) apply to current patients? Unfortunately, their analysis describes predictors for ischemia over the entire 20042017 period without accounting for the year of testing. Predictors of inducible ischemia in patients undergoing SPECT between 2015 and 2017 (or a more recent cohort) probably would be more applicable to current populations compared to predictors based on earlier data. For example, Rozanski and colleagues previously demonstrated that $12.7 \%$ of patients tested between 1991 and 1995 had typical angina compared to only $1.9 \%$ of patients between 2006 and $2009 .^{3}$ If typical angina is declining over time, does it still predict SPECT ischemia in current practice? This issue is not addressed by this study.

Our second concern is the evolving management of CAD, myocardial infarction, hyperlipidemia, and hypertension over the past 30 years. In the widely cited nuclear substudy of the COURAGE trial, optimal medical therapy was associated with improved myocardial perfusion as assessed by summed stress score (SSS). ${ }^{6}$ Anti-hyperlipidemia therapy has also been shown to decrease SSS by SPECT. ${ }^{7}$ Risk factor detection and treatment have intensified recently with more aggressive management targets as directed by national 
guidelines. ${ }^{8,9}$ Risk factor modification including hypertension control and the use of statins are now more likely in patients who are candidates for SPECT, reducing the prevalence of inducible ischemia. Unfortunately, details of medical therapy at the time of SPECT are not presented here, so the analysis could not consider the impact of optimal medical therapy. This omission is common elsewhere in the literature; virtually all databases constructed prior to 2008 did not capture these details, as the potential impact of medical therapy on SPECT results had not yet been recognized.

A third concern is that not all of the three strong predictors would be readily available to the ordering provider in everyday practice. Measurement of LVEF would not always be available prior to SPECT, especially in patients without a known history of CAD. A more practical alternative to LVEF would be a normal rest ECG. We have previously demonstrated the low yield of echocardiography in patients with chest pain and a normal rest ECG, as more than $95 \%$ of these patients had completely normal echocardiograms. ${ }^{10}$ In the multivariable analysis, it may have been more clinically meaningful to include a normal ECG (but not include LVEF) in order to better reflect information that would be readily available to the clinician before ordering SPECT.

These limitations are certainly not unique to this study, as they are common throughout the literature. The authors correctly acknowledge that this single-center study is potentially subject to selection bias and therefore requires duplication elsewhere. We wholeheartedly agree with their comments regarding the limitations of the traditional Diamond-Forrester method for estimating pre-test probability. ${ }^{11}$ Since those estimates were first developed, clinical practice has changed. The patients who are currently undergoing cardiac stress testing are older, more likely female, and less likely to have typical angina. More importantly, the underlying prevalence of CAD appears to be decreasing. Both of these changes create a "moving target" that limits the application of any retrospective analysis of previously studied patients to current practice.

The revision of pre-test probability estimates has proven to be both difficult and controversial. We have addressed that issue elsewhere. ${ }^{11}$ The recently published European Society of Cardiology estimates, although widely cited, are based on three prior publications with substantial selection bias. ${ }^{12}$ For example, in one of these studies, symptoms had little effect on pre-test probability estimates. The most reliable estimates appear to be the European CAD consortium estimates published by Genders et al in 2011. ${ }^{13}$ These estimates reported a lower prevalence of CAD in women, even when they have typical angina. When these estimates were tested for the presence of obstructive $\mathrm{CAD}$ on computed tomography angiography (CTA) in the SCOT-HEART study, they performed reasonably well. The addition of risk factors identified more low probability patients, but at a cost of more misclassifications. ${ }^{14}$

Why has CAD declined? Presumably, the prevalence of CAD at a given age has declined due to lifestyle changes and the resulting improvement in risk factor profiles. The Centers for Disease Control reported a $44 \%$ reduction in cardiovascular mortality between 1980 and 2000 due to population improvements in risk factors without treatment. ${ }^{15}$ These improvements have also been associated with a decrease in the prevalence of obstructive CAD. In a population-based study from Mayo Clinic that considered both invasive angiography and CTA, the age- and sex-adjusted rate of obstructive CAD declined by $30 \%$ between 2000 and $2018 .^{16}$ Thus, we must recognize that studies of SPECT, and of other approaches to the diagnosis of CAD, are limited in their accuracy due to this "moving target."

The appropriate use criteria (AUC) and the Choosing Wisely Campaign have both tried to reduce unnecessary testing; current reimbursement policies, which reward unnecessary testing, have limited these efforts. The PROMISE trial developed a "minimalrisk" tool and reported that $25 \%$ of patients with stable chest pain in North America had normal coronary arteries and no long-term cardiac events. ${ }^{17}$ A subsequent "real-world" application of that tool at Duke University Medical Center found that $17 \%$ of patients undergoing cardiac catherization for stable chest pain met the criteria for "minimal risk" and had normal coronary arteries. $^{18}$ Short-term outcomes in these patients appeared to be favorable. Unfortunately, the generalizability of such tools is open to question. When the PROMISE tool, originally developed in a North American population, was tested in the SCOT-HEART population, its calibration was poor. The same variables were meaningful, but the coefficients had to be re-calculated to create a new model. ${ }^{19}$ Therefore, the assumption that risk tools are generalizable is very questionable, even when they are applied to different populations in the same time frame. Their accuracy will likely be even worse if they are applied to different populations in different time periods, as the characteristics of one of the populations will likely have changed over time. The principles for the rigorous application of predictive tools have been defined ${ }^{20}$; we would suggest that they are infrequently followed due to their difficulty.

Despite the challenge of studies such as the one by Rozanski et al, they must and should be done to better educate all of us regarding the performance of SPECT studies so that we can improve their utility and cost- 
effectiveness. Despite its limitations, this study certainly adds to our understanding in this important area.

\section{Disclosures}

The authors report no potential conflict of interest.

\section{References}

1. Jouni H, Askew JW, Crusan DJ, Miller TD, Gibbons RJ. Temporal trends of single-photon emission computed tomography myocardial perfusion imaging in patients without prior coronary artery disease: A 22-year experience at a tertiary academic medical center. Am Heart J 2016;176:127-33.

2. Jouni H, Askew JW, Crusan DJ, Miller TD, Gibbons RJ. Temporal trends of single-photon emission computed tomography myocardial perfusion imaging in patients with coronary artery disease: A 22-year experience from a Tertiary Academic Medical Center. Circ Cardiovasc Imaging 2017;10(7).

3. Rozanski A, Gransar H, Hayes SW, Min J, Friedman JD, Thomson LE, et al. Temporal trends in the frequency of inducible myocardial ischemia during cardiac stress testing: 1991 to 2009. J Am Coll Cardiol 2013;61:1054-65.

4. Rozanski A, Miller RH, Han D, Gransar H, Slomka PJ, Dey D, et al. The prevalence and predictors of inducible myocardial ischemia among patients referred for radionuclide stress testing. JNC 2021;TBD.

5. Duvall WL, Rai M, Ahlberg AW, O'Sullivan DM, Henzlova MJ. A multi-center assessment of the temporal trends in myocardial perfusion imaging. J Nucl Cardiol 2015;22:539-51.

6. Shaw LJ, Berman DS, Maron DJ, Mancini GB, Hayes SW, Hartigan PM, et al. Optimal medical therapy with or without percutaneous coronary intervention to reduce ischemic burden: Results from the Clinical Outcomes Utilizing Revascularization and Aggressive Drug Evaluation (COURAGE) trial nuclear substudy. Circulation 2008;117:1283-91.

7. Schwartz RG, Pearson TA, Kalaria VG, Mackin ML, Williford DJ, Awasthi A, et al. Prospective serial evaluation of myocardial perfusion and lipids during the first six months of pravastatin therapy: Coronary artery disease regression single photon emission computed tomography monitoring trial. J Am Coll Cardiol 2003;42:600-10.

8. Grundy SM, Stone NJ, Bailey AL, Beam C, Birtcher KK, Blumenthal RS, et al. 2018 AHA/ACC/AACVPR/AAPA/ABC/ ACPM/ADA/AGS/APhA/ASPC/NLA/PCNA guideline on the management of blood cholesterol: executive summary: A Report of the American College of Cardiology/American Heart Association Task Force on Clinical Practice Guidelines. J Am Coll Cardiol 2019;73:3168-209.

9. Whelton PK, Carey RM, Aronow WS, Casey DE, Collins KJ, Dennison Himmelfarb C, et al. 2017 ACC/AHA/AAPA/ABC/ ACPM/AGS/APhA/ASH/ASPC/NMA/PCNA guideline for the prevention, detection, evaluation, and management of high blood pressure in adults: A Report of the American College of Cardiology/American Heart Association Task Force on Clinical Practice Guidelines. Hypertension 2018;71:e13-115.

10. Gibbons RJ, Carryer D, Liu H, Brady PA, Askew JW, Hodge D, et al. Use of echocardiography in outpatients with chest pain and normal resting electrocardiograms referred to Mayo Clinic Rochester. Am Heart J 2018;196:49-55.

11. Gibbons RJ, Miller TD. Declining accuracy of the traditional diamond-Forrester estimates of pretest probability of coronary artery disease: Time for new methods. JAMA Intern Med 2021;181:579-80.

12. Knuuti J, Wijns W, Saraste A, Capodanno D, Barbato E, FunckBrentano C, et al. 2019 ESC Guidelines for the diagnosis and management of chronic coronary syndromes. Eur Heart $\mathrm{J}$ 2020;41:407-77.

13. Genders TS, Steyerberg EW, Alkadhi H, Leschka S, Desbiolles L, Nieman K, et al. A clinical prediction rule for the diagnosis of coronary artery disease: Validation, updating, and extension. Eur Heart J 2011;32:1316-30.

14. Baskaran L, Danad I, Gransar H, Hartaigh BO, Schulman-Marcus $\mathrm{J}$, Lin FY, et al. A comparison of the updated diamond-Forrester, CAD consortium, and CONFIRM history-based risk scores for predicting obstructive coronary artery disease in patients with stable chest pain: The SCOT-HEART coronary CTA cohort. JACC Cardiovasc Imaging 2019;12:1392-400.

15. Ford ES, Ajani UA, Croft JB, Critchley JA, Labarthe DR, Kottke TE, et al. Explaining the decrease in U.S. deaths from coronary disease, 1980-2000. N Engl J Med 2007;356:2388-98.

16. Gerber Y, Gibbons RJ, Weston SA, Fabbri M, Herrmann J, Manemann SM, et al. Coronary disease surveillance in the community: Angiography and revascularization. J Am Heart Assoc 2020;9:e015231.

17. Fordyce CB, Douglas PS, Roberts RS, Hoffmann U, Al-Khalidi HR, Patel MR, et al. Identification of patients with stable chest pain deriving minimal value from noninvasive testing: The PROMISE minimal-risk tool, a secondary analysis of a randomized clinical trial. JAMA Cardiol 2017;2:400-8.

18. Nanna MG, Wang TY, Chiswell K, Sun JL, Vemulapalli S, Hoffmann U, et al. Estimating the real-world performance of the PROMISE minimal-risk tool. Am Heart J 2021;239:100-9.

19. Adamson PD, Fordyce CB, McAllister DA, Udelson JE, Douglas PS, Newby DE. Identification of patients with stable chest pain deriving minimal value from coronary computed tomography angiography: An external validation of the PROMISE minimalrisk tool. Int J Cardiol 2018;252:31-4.

20. Pencina MJ, Goldstein BA, D'Agostino RB. Prediction modelsDevelopment, evaluation, and clinical application. N Engl J Med 2020;382:1583-6.

Publisher's Note Springer Nature remains neutral with regard to jurisdictional claims in published maps and institutional affiliations. 